\title{
Sticky Impact - Building Teaching and Learning on Performative Practice
}

\author{
Fionn Woodhouse
}

The Workshop - Based on research into the long term impact of participation in drama-based workshops focusing on goal setting, this workshop hoped to investigate how we can make the performative more 'sticky'. How can performative approaches be combined with other modes of learning, methods of teaching to allow students and teachers new pathways to learning?

Taking inspiration from the symposium question - 'Wherein exactly lies the benefit of applying performative approaches?' - this workshop asked participants to play with, and reflect on, different approaches to performativity in the classroom/seminar/lecture setting, with the aim of allowing participants to create 'sticky impact' - impact from participation that stays with the learner.

The workshop was a practice based session with a focus on performative exercises and activities that have been/could be used in a teaching and learning context.

The session began with an initial introduction via slides outlining the workshops themes, theoretical contexts, and the practice based nature of the workshop. Following this the workshop section began with participants engaging in a series of performative drama activities that allowed participants to suggest/influence/lead how the activity developed. An initial 'warm up' activity of a name game involved participants individually saying their name followed a sound and movement that captured their journey to the workshop. The name, sound and movement are then repeated by the group. As the facilitator I briefly outline the activity and demonstrate a range of potential engagements, essentially 'showing (potential) doing'. Participants then performatively engage with the activity individually and as a group, showing and responding to each other. Briefly introducing ideas around non-verbal communication, self-expression and perception this activity primarily functioned a 'get to know you' role whilst allowing the facilitator to hand control over to the group immediately.

This was followed by a number of activities that asked participants to adapt common drama exercises (such as Zip, Zap, Boing) for use in a new teaching and learning context. Through the process of adapting the activities for use in a range of different teaching settings and subjects, participants engaged in refining the performative processes to include specific learning outcomes. 
These ranged from language acquisition to spatial awareness to understanding political/state structures and geography.

The range of ways participants adapted the activites and probed the potential 'stickness' of the learning processes during the workshop, allowed spaces for reflection on how the process of adapting in itself creates 'sticky impact'.

Understanding the Performative - The experience of taking part in workshops such as 'Inszenierung von Lernprozessen' with Ulrike Jaeger and Bärbel Jogschies allowed my understanding of the range of work encompassed by the 'performative' umbrella term to expand in a very concrete way. The workshops, along with talks such as Michaela Sambanis' and Maik Walter's 'Und Action! Vom Hirn ins Herz bis in die Füße', gave insight into both the practical uses, and the expanding theoretical foundations, upon which learning can be built. The symposium introduced scholars, practitioners and educators to concrete examples of performative practice and made particpants aware of new approaches to building performative teaching and learning across a range of institutions.

Taking the Stage - Whilst there are some opportunities to spread the word of performative teaching and learning at local levels within Irish universities, and there is increasing awareness of the need for physical spaces that can accommodate this type of teaching, much more needs to be done to increase the awareness of the range of applications. There are still widely held but narrow views across academia as to what the performative does in the classroom and what it could mean for engaging students. Recent strategic planning has included copious references to 'creativity', 'creative endeavour' and 'collaborative enquiry' all of which are at the core of performative pedagogy however there is much less substance as to what these terms may mean at local levels. Events such as the Scenrio Symposia and Conferences which build awareness of current research such as that conducted by Michaela Sambanis, and allow insights into how other institutions are developing performative teaching, learning, and research are very important as they offer opportunities for a distillation of thinking across the field, the results of which can be seen in the 'Recommendations for Promoting a Perfromative Teaching, Learning, and Research Culture in Higher Education'. These set of Recommendations bring together in a clear and concise manner the current understanding of the field and the steps needed to promote further engagement across higher education. With the Recommendations as a 'script', the stage is set for a performative turn! 